\title{
Editorial
}

\section{Control of Vancomycin-Resistant Enterococci: It Is Important, It Is Possible, and It Is Cost-Effective}

\author{
C. Glen Mayhall, MD
}

Clinical isolates of enterococci resistant to vancomycin were first detected in Europe in 1986 and reported in 1988.1,2 By 1989, vancomycin-resistant enterococci (VRE) were being recovered from cultures of clinical specimens in the United States, and by 1993, VRE made up 7.9\% of nosocomial enterococci reported by hospitals in the National Nosocomial Infections Surveillance System of the Centers for Disease Control and Prevention (CDC) ${ }^{3}$ In the past decade, VRE have spread throughout the country, and the only places where they have not been found are probably in places where no one has looked.

Although work remains to be done, the epidemiology of nosocomial VRE is well understood. The most important reservoir is the colonized gastrointestinal tracts of patients. VRE are transmitted between patients by the contaminated hands and clothes of healthcare workers and perhaps indirectly from contamination of the environment, particularly when at least one patient has diarrhea.,5 VRE have been shown to survive for prolonged periods of time in the environment, and an outbreak in a burn unit recurred after 5 weeks from an electrocardiogram lead that had been contaminated by a colonized patient 38 days before VRE was cultured from the lead on the second patient. ${ }^{6-8}$ Risk factors for acquisition of VRE include treatment with vancomycin ${ }^{9,10}$ and cephalosporins, ${ }^{11,12}$ receipt of enteral feedings, ${ }^{11,13}$ sucralfate, ${ }^{13}$ or antacids, ${ }^{8,10}$ colonization pressure, ${ }^{11}$ and proximity to previously unisolated patients with VRE. ${ }^{14}$

On the basis of the epidemiology of VRE, in 1995 the Hospital Infection Control Practices Advisory Committee
(HICPAC) of the CDC published recommendations for preventing the spread of vancomycin resistance. ${ }^{15}$ The major elements of the recommendations include control of vancomycin use, early detection of VRE in patient populations, and rectal surveillance cultures to detect patients colonized with VRE followed by contact isolation of colonized patients using gowns and gloves. Since their publication, these recommendations have been adopted by many institutions for the control of VRE. However, some investigators have suggested that rectal surveillance cultures are costly and time consuming ${ }^{16-18}$ or that the control measures are ineffective. ${ }^{19,20}$ VRE apparently were endemic in the hospitals of the authors of three of these publications. ${ }^{16,17,19}$ In two of the publications, the authors questioned whether the HICPAC/CDC recommendations for control of VRE would likely be effective in preventing the spread of these resistant microorganisms. ${ }^{19,20}$ One group of investigators mentioned that a similar infection control strategy failed to control methicillin-resistant Staphylococcus aureus and therefore would be unlikely to control VRE. ${ }^{20}$

It is unfortunate that such publications may have a negative effect on hospital epidemiologists and infection control practitioners faced with the responsibility for control of VRE in their hospitals. This is particularly unfortunate given the number of published accounts of control of VRE using the control measures recommended by HICPAC/CDC whether these measures were applied before or after publication of the HICPAC/CDC recommendations. The number of these publications greatly exceeds the number of published reports of failure of the 
measures recommended by $\mathrm{HICPAC} / \mathrm{CDC}{ }^{4,8,14,21-30}$ The $\mathrm{HICPAC/CDC}$ recommendations have been effective in controlling VRE in many different clinical settings, including a burn unit, ${ }^{8}$ a cardiothoracic intensive care unit, ${ }^{21}$ an oncology intensive care unit, ${ }^{29}$ a surgical intensive care unit, ${ }^{28}$ a neonatal intensive care unit, ${ }^{30}$ pediatric patients, ${ }^{22}$ a renal unit, ${ }^{24}$ an adult oncology unit, ${ }^{26}$ and a pediatric oncology unit. ${ }^{25}$ The recommendations have been successfully applied in the United States, $4,8,14,21-23,27-30$ the United Kingdom, ${ }^{24,25}$ and South Africa. ${ }^{26}$ The recommendations proved effective when used in a country with limited resources. ${ }^{26}$ The recommendations worked well when a polymerase chain reaction assay was used to detect VRE in rectal specimens in place of cultures. ${ }^{31}$ In their most extensive test, the recommendations were effective in controlling VRE in 32 healthcare facilities in the Siouxland region of Iowa, Nebraska, and South Dakota. ${ }^{27}$ Seldom has any set of recommendations been so thoroughly tested and proven. They are effective when they are applied as designed.

Even if the HICPAC/CDC recommendations are effective in controlling the spread of VRE, the argument is often made that it is not necessary to control VRE. After all, VRE are only normal flora with vancomycin resistance. They are not invasive and seldom cause serious disease. However, the preponderance of studies on VRE bacteremia suggest otherwise. Four studies showed a significant relationship between VRE bacteremia and a fatal outcome whether patients with VRE bacteremia were compared with patients with no bacteremia ${ }^{32}$ or with patients with bacteremia caused by vancomycin-sensitive enterococci ${ }^{33-35}$ In two studies, VRE bacteremia was noted to often be persistent ${ }^{36,37}$ and refractory to treatment. ${ }^{37}$ In one study, the cost of bacteremia due to VRE was more than $\$ 27,000$ higher than the cost of bacteremia due to vancomycin-sensitive enterococci ${ }^{33}$ Regardless of whether clinicians believe that VRE is a significant pathogen, all would feel compelled to treat a confirmed VRE bacteremia. Therapy will essentially always be with one of two expensive antimicrobial agents-quinupristin/dalfopristin or linezolid.

Although the effectiveness of the HICPAC/CDC recommendations in controlling the spread of VRE is already well documented, two articles in this issue of Infection Control and Hospital Epidemiology make significant contributions in two areas of VRE control. In the first article, Srinivasan et al. provide evidence that gowns and gloves provide significantly more protection than gloves alone against transmission of VRE, lending further support to the recommendations for the use of gowns and gloves in the HICPAC/CDC recommendations. ${ }^{38}$ These authors examined the effects of gowns and gloves for 3 months in a medical intensive care unit followed by a 3-month period during which only gloves were used. Patients enrolled in the two periods did not differ significantly regarding age, gender, race, treatment with antibiotics, or underlying diseases. The VRE acquisition rate was 1.80 cases per 100 days at risk during the gown and gloves period versus 3.78 cases per 100 days at risk during the gloves only period.
Multivariate analysis using a proportional hazards model revealed a significantly higher rate of transmission of VRE during the gloves only period compared with the gown and gloves period (hazard ratio, 2.5; 95\% confidence interval, 1.2 to $5.3 ; P=.02$ ).

The findings in this study differ from those of a study previously published by Slaughter et al. in which there was no observed difference in transmission of VRE in a medical intensive care unit when gloves alone versus gowns and gloves were used to prevent the spread of VRE. ${ }^{13}$ Srinivasan et al. suggest that the differences in the findings of the two studies may be due to the low prevalence of environmental contamination $(6.3 \%)$ in the medical intensive care unit studied by Slaughter et al. and also due to differences in study design. In the latter study, the two populations studied (gloves versus gowns and gloves) were present in the medical intensive care unit at the same time, in contrast to the study of Srinivasan et al. in which the two populations were studied sequentially. Other investigators should try to reproduce these results in similarly designed studies but with a larger number of patients and more extensive efforts to control for confounding due to use of nasoenteric tubes, enteric feeding, medications that effect gastrointestinal tract function, nurse-patient ratios, degree of environmental contamination, and measurement of compliance with gown and gloves use.

In another article published in this issue, Muto et al. provide evidence that control of VRE using the $\mathrm{HICPAC} / \mathrm{CDC}$ recommendations is not only possible, it is cost-effective. ${ }^{39}$ These investigators did a comparative study using their institution and another university hospital in the same region. The latter hospital was chosen because of similarities with the authors' hospital regarding number of beds, number of admissions, and services offered. During the 2 years used for comparison, 1 VRE bacteremia occurred at the authors' hospital and 29 at the other hospital. After careful estimates of the costs for additional medical care for VRE bacteremias and of the costs of applying the HICPAC/CDC recommendations for control of VRE, the authors observed that the costs of the bacteremias were threefold greater than the costs of the control program. Because of the magnitude of the differences, even if there were errors in some of the authors' estimates, it seems likely that control would still be less expensive than treatment of bacteremias.

Thus, not only has it been documented that application of the HICPAC/CDC recommendations for the control of VRE is effective, but now data from two articles in this issue support a key recommendation in the HICPAC/CDC recommendations (ie, use of both gowns and gloves for barrier precautions) and indicate that use of the recommendations is also cost-effective.

If all of the above is not reason enough to adopt the HICPAC/CDC recommendations for control of VRE, the possibility that VRE may transfer genetic elements that code for vancomycin resistance to strains of methicillinresistant $S$. aureus, present at the same time in patients colonized with VRE, should also provide a strong impetus for 
implementation of these control measures. The vanA gene has been transferred in vitro from Enterococcus faecalis to S. aureus, ${ }^{40}$ and was observed for the first time in a clinical isolate of $S$. aureus in Michigan in June 2002. ${ }^{41}$ This isolate was found in a patient who had had methicillin-resistant $S$. aureus and VRE, the situation in which it had been anticipated that this might occur.

There has been a certain malaise in the hospital epidemiology and infection control community about the need to control the spread of VRE. This seems to stem from a belief that VRE are not pathogenic, that it is impossible to prevent spread in hospital populations, and that control of VRE is not cost-effective. There are data from several studies that document the pathogenicity of VRE and a large number of studies showing that VRE can be controlled by use of the HICPAC/CDC recommendations. Now there are data that further document the cost-effectiveness of control.

It is time for a renewed effort to control VRE. It is important, it is possible, and it is cost-effective. These recommendations should be strongly supported by the Society for Healthcare Epidemiology of American (SHEA) and the Association for Professionals in Infection Control and Epidemiology (APIC). In the absence of new data indicating that the control measures included in the 1995 HICPAC recommendations are ineffective (when used as described in the recommendations), the major elements should remain unchanged when and if the recommendations are revised. Because gowns and gloves are important for effective control, active surveillance cultures become critically necessary. This is because one cannot know when to use these barriers unless colonized patients are identified. In one recent study, 95\% of VREcolonized patients never had a positive clinical microbiology culture. ${ }^{42}$ For this reason, a program of active surveillance cultures for patients at risk of carriage must be recommended to guide the use of contact precautions in every healthcare facility.

\section{REFERENCES}

1. Uttley AHC, Collins $\mathrm{CH}$, Naidoo J, George RC. Vancomycin-resistant enterococci. Lancet 1988;1:57-58.

2. Leclercq R, Derlot E, Duval J, Courvalin P. Plasmid-mediated resistance to vancomycin and teicoplanin in Enterococcus faecium. N Engl J Med 1988;319:157-161.

3. Centers for Disease Control and Prevention. Nosocomial enterococci resistant to vancomycin: United States, 1989-1993. MMWR 1993;42:597599.

4. Boyce JM, Opal SM, Chow JW, et al. Outbreak of multidrug-resistant Enterococcus faecium with transferable van B class vancomycin resistance. I Clin Microbiol 1994;32:1148-1153.

5. Bonten MJM, Hayden MK, Nathan C, et al. Epidemiology of colonization of patients and environment with vancomycin-resistant enterococci. Lancet 1996;348:1615-1619.

6. Noskin GA, Stosor V, Cooper I, Peterson LR. Recovery of vancomycinresistant enterococci on fingertips and environmental surfaces. Infect Control Hosp Epidemiol 1995;16:577-581.

7. Bonilla $\mathrm{HF}$, Zervos MJ, Kauffman CA. Long-term survival of vancomycin-resistant Enterococcus faecium on a contaminated surface. Infect Control Hosp Epidemiol 1996;17:770-772.

8. Falk PS, Winnike J, Woodmansee C, Desai M, Mayhall CG. Outbreak of vancomycin-resistant enterococci in a burn unit. Infect Control Hosp Epidemiol 2000;21:575-582.

9. D'Agata EMC, Green WK, Schulman G, Li H, Tang Y-W, Schaffner W. Vancomycin-resistant enterococci among chronic hemodialysis patients: a prospective study of acquisition. Clin Infect Dis 2001;32:2329.

10. Cetinkaya Y, Falk PS, Mayhall CG. Effect of gastrointestinal bleeding and oral medications on acquisition of vancomycin-resistant Enterococcus faecium in hospitalized patients. Clin Infect Dis. In press.

11. Bonten MJM, Slaughter S, Ambergen AW, et al. The role of "colonization pressure" in the spread of vancomycin-resistant enterococci: an important infection control variable. Arch Intern Med 1998;158:11271132.

12. Loeb M, Salama S, Armstrong-Evans M, Capretta G, Olde J. A case-control study to detect modifiable risk factors for colonization with vancomycin-resistant enterococci. Infect Control Hosp Epidemiol 1999;20:760-763.

13. Slaughter $S$, Hayden MK, Nathan $C$, et al. A comparison of the effect of universal use of gloves and gowns with that of glove use alone on acquisition of vancomycin-resistant enterococci in a medical intensive care unit. Ann Intern Med 1996;125:448-456.

14. Byers KE, Anglim AM, Anneski CJ, et al. A hospital epidemic of vancomycin-resistant Enterococcus: risk factors and control. Infect Control Hosp Epidemiol 2001;22:140-147.

15. Hospital Infection Control Practices Advisory Committee Recommendations for preventing the spread of vancomycin resistance. Infect Control Hosp Epidemiol 1995;16:105-113.

16. Wells CL, Juni BA, Cameron SB, et al. Stool carriage, clinical isolation, and mortality during an outbreak of vancomycin-resistant enterococci in hospitalized medical and/or surgical patients. Clin Infect Dis 1995;21:4550.

17. Lai KK, Kelley AL, Melvin ZS, Belliveau PP, Fontecchio SA. Failure to eradicate vancomycin-resistant enterococci in a university hospital and the cost of barrier precautions. Infect Control Hosp Epidemiol 1998;19:647-652.

18. Zuckerman RA, Steel L, Venezia RA, Tobin EH. Undetected vancomycinresistant Enterococcus in surgical intensive care unit patients. Infect Control Hosp Epidemiol 1999;20:685-686.

19. Morris JG Jr, Shay DK, Hebden JN, et al. Enterococci resistant to multiple antimicrobial agents, including vancomycin: establishment of endemicity in a university medical center. Ann Intern Med 1995;123:250259.

20. Goetz AM, Rihs JD, Wagener MM, Muder RR. Infection and colonization with vancomycin-resistant Enterococcus faecium in an acute care Veterans Affairs Medical Center: a 2-year survey. Am I Infect Control 1998;26:558-562.

21. Karanfil LV, Murphy M, Josephson A, et al. A cluster of vancomycinresistant Enterococcus faecium in an intensive care unit. Infect Control Hosp Epidemiol 1992;13:195-200.

22. Rubin LG, Tucci V, Cercenado E, Eliopoulos G, Isenberg HD. Vancomycin-resistant Enterococcus faecium in hospitalized children. Infect Control Hosp Epidemiol 1992;13:700-705.

23. Boyce JM, Mermel LA, Zervos MJ, et al. Controlling vancomycin-resistant enterococci. Infect Control Hosp Epidemiol 1995;16:634-637.

24. Brown AR, Amyes SGB, Paton R, et al. Epidemiology and control of vancomycin-resistant enterococci (VRE) in a renal unit. $J$ Hosp Infect $1998 ; 40: 115-124$

25. Nourse C, Byrne C, Murphy H, Kaufmann ME, Clarke A, Butler K Eradication of vancomycin-resistant Enterococcus faecium from a paediatric oncology unit and prevalence of colonization in hospitalized and community-based children. Epidemiol Infect 2000;124:53-59.

26. McCarthy KM, Van Nierop W, Duse A, et al. Control of an outbreak of vancomycin-resistant Enterococcus faecium in an oncology ward in South Africa: effective use of limited resources. J Hosp Infect 2000;44:294-300.

27. Ostrowsky BE, Trick WE, Sohn $\mathrm{AH}$, et al. Control of vancomycin-resistant Enterococcus in health care facilities in a region. $N$ Engl $J$ Med 2001;344:1427-1433.

28. Hendrix CW, Hammond JMJ, Swoboda SM, et al. Surveillance strategies and impact of vancomycin-resistant enterococcal colonization and infection in critically ill patients. Ann Surg 2001;233:259-265.

29. Hanna H, Umphrey J, Tarrand J, Mendoza M, Raad I. Management of an outbreak of vancomycin-resistant enterococci in the medical intensive care unit of a cancer center. Infect Control Hosp Epidemiol 2001;22:217 219.

30. Rupp ME, Marion N, Fey PD, et al. Outbreak of vancomycin-resistant Enterococcus faecium in a neonatal intensive care unit. Infect Control Hosp Epidemiol 2001;22:301-303.

31. Roger M, St-Antoine P, Coutlee F. Vancomycin-resistant enterococci in health care facilities. $N$ Engl J Med 2001;345:768-769.

32. Edmond MB, Ober JF, Dawson JD, Weinbaum DL, Wenzel RP. Vancomycin-resistant enterococcal bacteremia: natural history and attributable mortality. Clin Infect Dis 1996;23:1234-1239.

33. Stosor V, Peterson LR, Postelnick M, Noskin GA Enterococcus faecium bacteremia: does vancomycin resistance make a difference? Arch Intern 
Med 1998;158:522-527.

34. Vergis EN, Hayden MK, Chow JW, et al. Determinants of vancomycin resistance and mortality rates in enterococcal bacteremia: a prospective multicenter study. Ann Intern Med 2001;135:484-492.

35. Linden PK, Pasculle AW, Manez R, et al. Differences in outcomes for patients with bacteremia due to vancomycin-resistant Enterococcus faecium or vancomycin-susceptible $E$. faecium. Clin Infect Dis 1996;22:663-670

36. Montecalvo MA, Shay DK, Patel P, et al. Bloodstream infections with vancomycin-resistant enterococci. Arch Intern Med 1996;156:14581462.

37. Montecalvo MA, Horowitz $\mathrm{H}$, Gedris $\mathrm{C}$, et al. Outbreak of vancomycin-, ampicillin-, and aminoglycoside-resistant Enterococcus faecium bacteremia in an adult oncology unit. Antimicrob Agents Chemother 1994;38:1363-1367.

38. Srinivasan A, Song X, Ross T, Merz W, Brower R, Perl TM. A prospective study to determine whether cover gowns in addition to gloves decrease nosocomial transmission of vancomycin-resistant enterococ$c i$ in an intensive care unit. Infect Control Hosp Epidemiol 2002;23:424428.

39. Muto CA, Giannetta ET, Durbin LJ, Simonton BM, Farr BM. Costeffectiveness of perirectal surveillance cultures for controlling vancomycin-resistant Enterococcus. Infect Control Hosp Epidemiol 2002;23:429-435.

40. Noble WC, Virani Z, Cree R. Co-transfer of vancomycin and other resistance genes from Enterococcus faecalis NCTC12201 to Staphylococcus aureus. FEMS Microbiol Lett 1992;72:195-198.

41. Centers for Disease Control and Prevention. Staphylococcus aureus resistant to vancomycin: United States, 2002. MMWR 2002;51:565-567.

42. Calfee DP, Giannetta ET, Farr BM. Effective control of VRE colonization using CDC recommendations for detection and isolation. Presented at the 38th Annual Meeting of the Infectious Diseases Society of America; September 7-10, 2000; New Orleans, LA. Abstract 21. 